\title{
Does COVID-19 Cause New-Onset Diabetes or Unmask Pre-Existing Diabetes?
}

\section{Mikhail N* and Wali S}

Endocrinology Division, Department of Medicine, Olive View-UCLA Medical Center, David-Geffen-UCLA Medical School, CA, USA

"Correspondence: Nasser Mikhail, Endocrinology Division, Department of Medicine, Olive View-UCLA Medical Center, David-Geffen-UCLA Medical School, CA, USA

Received on 04 February 2021; Accepted on 13 February 2021; Published on 16 February 2021

Copyright (c) 2021 Mikhail N, et al. This is an open access article and is distributed under the Creative Commons Attribution License, which permits unrestricted use, distribution, and reproduction in any medium, provided the original work is properly cited.

\begin{abstract}
Background: Many cases of newly diagnosed diabetes were reported in association with coronavirus 2019 (COVID-19) caused by the severe acute respiratory syndrome-associated coronavirus-2 (SARS-CoV-2).

Objective: To clarify whether COVID-19 triggers new diabetes or unmask pre-existing undiagnosed diabetes.

Methods: PubMed search of literature up to February 3, 2021. Search terms included diabetes, COVID-19, diagnosis, hemoglobin Alc (HbAlc), diabetic ketoacidosis, diabetes ketoacidosis, pancreatitis. Case reports, case series, retrospective studies, reviews, and pertinent in-vitro investigations were reviewed.

Results: Retrospective studies and case series suggest that COVID-19 can worsen diabetes control and precipitate hyperglycemic crises in patients admitted to the hospital. Majority of these patients had pre-existing undiagnosed type 2 diabetes as reflected by elevated HbAlc levels on admission. Many patients presenting with hyperglycemia and normal HbAlc levels may have transient stress hyperglycemia. This group of patients are misclassified as new-onset diabetes despite lack of patient follow-up after discharge. Only one case report of possible newonset diabetes described a patient with pre-diabetes who progressed to severe diabetes 6 weeks following COVID-19 pneumonia. Mechanisms of worsening glycemic control by COVID-19 infection include increased release of cytokines and insulin counter-regulatory hormones. Binding of SARS-CoV-2 to pancreatic $\beta$-cells and their subsequent destruction by the virus as another mechanism requires further studies.

Conclusion: COVID-19 infection commonly unmasks pre-existing diabetes. Follow-up of patients presenting with new-onset hyperglycemia after hospital discharge is essential to distinguish between stress hyperglycemia and new-onset diabetes.
\end{abstract}

Keywords: diabetes, COVID-19, new diagnosis, mechanisms, diabetic ketoacidosis 
Abbreviations: HbA1c: hemoglobin A1c; DKA: diabetic ketoacidosis; CRP: C-reactive protein; Il-6: interleukin 6; TNF-alpha: tumor necrosis factor alpha; ACE2: angiotensin-converting enzyme 2; TMPRSS2: transmembrane serine protease 2

\section{Introduction}

Accumulating data suggest that COVID-19, like other types of infection, can worsen hyperglycemia or precipitate hyperglycemic crises in patients with pre-existing diabetes [1]. In the meantime, many viral infections were known to trigger or cause type 1 diabetes, particularly enteroviruses (e.g., Coxsackie B1 virus), mumps, rubella and cytomegalovirus [2]. It is unclear whether COVID-19 worsens glycemic control or can by itself causes diabetes or both. Many studies described patients admitted to the hospital with "new-onset" diabetes [3, 4]. However, this description lacks precision for two reasons. First, hemoglobin A1c (HbA1c) levels were severely elevated in majority of patients in whom HbA1c measurement was performed [4]. Hence, these patients had pre-existing undiagnosed diabetes that was present for at least 2-3 months prior to hospital admission. Second, even if HbA1c values on admission are normal, this "new-onset" diabetes may represent a transient form of hyperglycemia called stress hyperglycemia that resolves after resolution of underlying condition [5]. Therefore, follow-up of COVID-19 patients presenting with hyperglycemia and normal $\mathrm{HbAlc}$ values is essential to distinguish between stress hyperglycemia and new-onset diabetes. Unfortunately, no follow-up was done to clarify this issue. Table 1 illustrates the distinction between new-onset diabetes, newly diagnosed diabetes, and stress hyperglycemia.

\begin{tabular}{|l|l|l|l|}
\hline & Newly diagnosed diabetes & New-onset diabetes & Stress hyperglycemia \\
\hline HbA1c levels & In diabetic range $\geq 6.5 \%$ & Below diabetic cutoff $<6.5 \%$ & Below diabetic cutoff $<6.5 \%$ \\
\hline Course & Long-lasting & Long-lasting & $\begin{array}{l}\text { Resolves after resolution of } \\
\text { stress after discharge from } \\
\text { hospital }\end{array}$ \\
\hline
\end{tabular}

Table 1: Distinction between causes of hyperglycemia at hospital admission. HbA1c: hemoglobin A1c.

\section{Frequency of Newly Diagnosed Diabetes in Association with COVID-19}

The frequency of newly diagnosed diabetes among patients admitted to the hospital with COVID-19 varies widely depending on patients' demographics, severity of COVID-19 and its treatment (particularly corticosteroids), and definition of newly diagnosed diabetes. In a meta-analysis of hospitalized patients with COVID-19 $(n=3,711)$, Satish et al. [6] defined newly diagnosed diabetes as fasting plasma glucose $\geq 7 \mathrm{mmol} / \mathrm{L}$ ( $126 \mathrm{mg} / \mathrm{dl})$ or random blood glucose of $\geq 11.1 \mathrm{mmol} / \mathrm{L}(200 \mathrm{mg} / \mathrm{dl})$, but with $\mathrm{HbA} 1 \mathrm{c}$ levels $<6.5 \%$. Based on this definition, they estimated that $14.4 \%$ (95\% CI, 5.9-25.8) of patients had newly diagnosed diabetes [6]. However, as mentioned above, this definition may include many patients with stress hyperglycemia which is temporary. The distinction between these 2 disease entities could not be done because none of the studies included in the meta-analysis performed any form of patient follow-up after hospital discharge [6]. In a retrospective study from Northern Italy, Fadini et al. [7] analyzed data of 413 patients admitted with COVID-19. They found that 21 patients (5\%) had newly diagnosed diabetes. The latter was defined by either one of the following 2 criteria: HbA1c value of $\geq 6.5 \%$, or a random glucose level of $\geq 11.1 \mathrm{mmol}(200 \mathrm{mg} / \mathrm{dl})$ [7]. Again, diagnosis of diabetes in hospitalized patients based solely on random glucose concentrations of $\geq 11.1$ $\mathrm{mmol}(200 \mathrm{mg} / \mathrm{dl})$ may be incorrect as it may include patients having transient stress hyperglycemia. 


\section{Frequency of Newly Diagnosed Diabetes Among Patients with COVID-19 Presenting with Diabetic Ketoacidosis}

Infection is a well-known precipitating factor for diabetic ketoacidosis (DKA) in patients with type 1 or type 2 diabetes [8]. Available evidence suggests that patients with COVID-19 presenting with DKA have underlying pre-existing diabetes. In the review of Pal et al. [4] of 19 studies of patients with COVID-19 presenting with DKA ( $\mathrm{n}=110)$, most of patients (77\%) had known pre-existing type 2 diabetes. In another 10 patients, diabetes was newly diagnosed [4]. However, 7 of these 10 patients had elevated HbA1c levels on admission ranging from 9.6-14.2\%, consistent with a history of previously undiagnosed diabetes. In the remaining 3 patients, HbA1c values were not measured. Yet, one of the 3 patients was morbidly obese with BMI $42.5 \mathrm{~kg} / \mathrm{m}^{2}$ [4]. Thus, it is likely that this patient suffered from undiagnosed type 2 diabetes [4].

\section{Type of newly diagnosed diabetes unmasked by COVID-19}

There are 3 lines of evidence that support the notion that newly diagnosed diabetes among patients admitted with COVID-19 is type 2 diabetes. This is expected because type 1 diabetes usually presents with acute symptoms of severe hyperglycemia or DKA leading to early diagnosis. The first line of evidence was derived from the study of Kuchay et al. [9] who followed 3 patients from India presenting with DKA and COVID-19 for 14 weeks. After 6 weeks of followup, these authors noticed decreased insulin requirements, and started to add oral anti-diabetic agents. After 14 weeks, diabetes was adequately controlled by 2 oral agents (metformin and DPP- 4 inhibitors) similar to patients with type 2 diabetes.

Second, in the few cases where markers of auto-immunity of type 1 diabetes were measured, these antibodies were found to be negative [9]. Thus, in 2 patients aged 30 and 34-year-old presenting with DKA and COVID-19, Kuchay et al. [9] demonstrated that antibodies of glutamic-acid decarboxylase (anti-GAD) were undetectable. Moreover, Hollstein et al. [10] from Germany reported a case of a 19-year old Caucasian man with COVID-19 and newly diagnosed diabetes presenting with DKA and extremely elevated HbA1c of $16.8 \%$. Auto-antibodies of GAD, islet cells, and zinc transporter 8 were all negative consistent with type 2 diabetes [10]. Third, another test that can differentiate between type 1 and type 2 diabetes is assessment of $\beta$-cell residual function by measurement of C-peptide levels, which are undetectable or extremely low in patients with type 1 diabetes. Thus, Ghosh et al. [11] from India did not found significant differences in C-peptide levels between newly diagnosed patients with diabetes who were COVID-19 positive $(\mathrm{n}=44) v s$. newly diagnosed diabetes who were COVID-19 negative $(\mathrm{n}=123)$. Of note, both groups of patients had similar mean HbA1c levels $10.2 \%$ [11].

Taken together, the above investigations showing achievement of glycemic control by oral agents upon patient followup, negative auto-antibodies of type 1 diabetes, and normal C-peptide levels all support the concept that type 2 diabetes, and not type 1, is the type of diabetes unmasked by COVID-19 [9-11].

\section{COVID-19 as a cause of "new-onset" type 2 diabetes}

To the best of the authors' knowledge, only one case report has provided some evidence that COVID-19 may cause new-onset type 2 diabetes [12]. Thus, Siddiki et al. [12] described a 58 years-old man from Jamaica who initially presented with COVID-19 pneumonia. His HbA1c level during this initial presentation was $6.1 \%$, i.e., in the prediabetes range [12]. Yet, 6 weeks after recovery from COVID-19 pneumonia, he presented with DKA. His biochemical profile was consistent with type 2 diabetes as evidenced by normal C-peptide levels, and negative antibodies against islet cell, zinc transporter 8, insulin, and weakly positive anti-GAD antibodies [12]. Interestingly, on this second admission, his polymerase chain reaction (PCR) was negative for COVID-19 and his HbA1c value was $10.8 \%$ implying that blood glucose levels started to rise rapidly after the initial COVID-19 pneumonia 6 weeks earlier [12]. 


\section{Potential Mechanisms by which COVID-19 Can Worsen Glycemic Control}

\section{Effect of cytokines on insulin resistance}

Many pro-inflammatory cytokines are released during the course of COVID-19 infection and correlate with disease severity such as C-reactive protein (CRP), interleukin 6 (Il-6), tumor necrosis factor alpha (TNF-alpha), D-dimers, pro-calcitonin and ferritin [13]. Accumulating evidence suggests that these cytokines may cause or worsen diabetes by increasing insulin resistance. Thus, in the prospective Women's Health Study, elevated levels of CRP and IL-6 predict the development of type 2 diabetes over a 4-year follow-up [14]. In the retrospective study of Martinez et al. [15] of 165 patients admitted for COVID-19 pneumonia, hyperglycemia was positively associated with the proinflammatory markers CRP and ferritin. Furthermore, Zhu et al. [16] compared levels of several pro-inflammatory markers between 2 groups of admitted COVID-19 patients: one group with well-controlled diabetes $(\mathrm{n}=282)$ (median blood glucose $6.4 \mathrm{mmol} / \mathrm{L}$, inter-quartile range 5.2-7.5) and the second groups with poorly controlled diabetes $(\mathrm{n}=$ 528) (median blood glucose $10.9 \mathrm{mmol} / \mathrm{L}$, inter-quartile range 7.6-14.3). They found that the following 3 markers were elevated in higher proportions of patients with poorly controlled diabetes than patients with well-controlled diabetes: $59.5 \%$ vs. $47.5 \%$ for CRP, $35.0 \%$ vs. $24.2 \%$ for procalcitonin, and $55.4 \%$ vs. $37.6 \%$ for D-dimers (P values were not reported) [16]. Interestingly, there is bidirectional relationship between cytokines and hyperglycemia. Thus, cytokines such as TNF-alpha and IL-1 may inhibit post-receptor insulin signaling and cause insulin resistance and hyperglycemia [5]. In turn, hyperglycemia stimulate more release of cytokines virtually setting up a vicious circle [5].

\section{Stress-induced release of insulin counter-regulatory hormones}

Stress such as infection, including COVID-19 infection, can lead to stress hyperglycemia. One main mechanism whereby stress increases blood glucose is by increasing release of insulin counter-regulatory hormones, namely cortisol, catecholamines and growth hormone [5]. These hormones impede insulin-mediated glucose uptake leading to hyperglycemia [5]. A second factor leading to stress-induced hyperglycemia is the release of cytokines as noted above. As mentioned earlier, stress hyperglycemia is a transient condition that resolves after resolution of underlying cause of stress [5].

\section{Binding of SARS-2 to pancreatic $\beta$-cells}

Entry of SARS-Cov-2 in its target cells requires binding of the viral spike (S) proteins to angiotensin-converting enzyme 2 (ACE2) receptors and presence of a serine protease called transmembrane serine protease 2 (TMPRSS2) in host cells [17]. TMPRSS2 is employed by SARS-CoV-2 for priming of the S protein, an essential step for viral entry into host cells [17]. Some studies have shown that ACE2 is expressed in pancreatic $\beta$-cells $[18,19]$. Therefore, binding of SARS-2-CoV-2 to $\beta$-cells could potentially lead to $\beta$-cell destruction, insulin deficiency and subsequent hyperglycemia. However, analysis of pancreatic sections from donors with and without diabetes by the group of Coate et al. [20] from USA did not reveal expression of ACE2 nor TMPRSS2 in $\beta$-cells. Therefore, direct binding of SARS2 -Cov-2 to pancreatic $\beta$-cells and their subsequent destruction requires further investigations.

\section{Acute pancreatitis}

Acute pancreatitis as a complication of COVID-19 uncommonly occurs and is usually mild [21]. In a series of 52 Chinese patients admitted for COVID-19 pneumonia, Wang et al. [21] reported that 9 subjects (17\%) had mild pancreatic injury defined as any elevation in serum levels of amylase or lipase. However, there was no significant difference in severity of hyperglycemia between patients with evidence of pancreatitis and those without pancreatitis [21]. Tollard et al. [22] described a French 32 years-old morbidly obese woman with COVID-19 who presented with acute pancreatitis and DKA. Meanwhile, this patient had evidence of baseline poorly controlled diabetes (HbA1c level of $13.1 \%$ ). Therefore, acute pancreatitis and uncontrolled type 2 diabetes, both may have contributed to DKA in this patient [22]. It should be emphasized that ACE2 was also shown to be the functional receptor of the predecessor of SARS-CoV-2 called SARS-CoV-1 [22]. Interestingly, in 2010, Yang et al. [22] described 20 patients admitted with 
SARS-CoV-1 pneumonia and with newly diagnosed diabetes (HbA1c levels not measured). After 3 years of followup, only 2 of these 20 patients had diabetes [22]. The authors hypothesized that SARS-CoV-1 had caused transient form of diabetes by binding and causing damage to pancreatic $\beta$-cells [22]. This hypothesis merits testing with respect to SARS-CoV-2.

\section{Conclusion and Current Needs}

Available data suggests that COVID-19 unmasks pre-existing diabetes rather than induces new-onset diabetes. To confirm this concept, future studies should provide two essential pieces of information. First, measurement of HbA1c levels is essential in any patient who presents with COVID-19 and hyperglycemia to distinguish between pre-existing diabetes in which $\mathrm{HbA} 1 \mathrm{c}$ levels are in diabetic range vs. new-onset diabetes and stress hyperglycemia. In the latter 2 conditions, HbA1c is below the diabetic HbA1c cutoff of $6.5 \%$. The second piece of information that distinguishes between new-onset of diabetes and stress hyperglycemia is the follow-up of patients few weeks after hospital discharge. In new-onset diabetes, hyperglycemia will be persistent, whereas in stress hyperglycemia, hyperglycemia will spontaneously normalize. To further clarify these critical issues, an international group of diabetes researchers have founded a global registry called "The CoviDIAB Project" [23]. Primary goals of this registry include verification of negative history of diabetes, and normal HbA1c levels on presentation with COVID-19. Other objectives include definition of the phenotype of diabetes and to confirm COVID-19 diagnosis [23]. In addition, the recently launched COVIDOM study of the University Hospital Schleswig-Holstein in Germany represents an epidemiological campaign with 10-year follow-up after SARS-Cov-2 infection. This prolonged follow-up should assess the long-term glycemic consequences of COVID-19 [10].

\section{Conflicts of Interest}

The authors do not have any conflict of interest to declare.

\section{References}

1. Mikhail N, Wali S. Hyperglycemic crises in patients with COVID-19. J Pathol Infect Dis. 2020;3(1):1-5.

2. Boddu SK, Aurangabadkar G, Kuchay MS. New onset diabetes, type 1 diabetes and COVID-19. Diabetes Metab Syndr. 2020;14(6):2211-217.

3. Smith SM, Boppana A, Traupman JA, et al. Impaired glucose metabolism in patients with diabetes, prediabetes, and obesity is associated with severe COVID-19. J Med Virol. 2020;93(1):409-15.

4. Pal R, Banerjee M, Yadav U, et al. Clinical profile and outcomes in COVID-19 patients with diabetic ketoacidosis: A systematic review of literature. Diab Metab Syndr. 2020;14(6):1563-569.

5. Dugna KM, Braithwaite SS, Preiser J-C. Stress hyperglycemia. Lancet. 2009;373(9677):1798-807.

6. Sathish T, Kapoor N, Cao Y, et al. Proportion of newly diagnosed diabetes in COVID-19 patients: A systematic review and meta-analysis. Diabetes Obes Metab. 2021;23(3):870-74.

7. Fadini GP, Morieri ML, Boscari F, et al. Newly-diagnosed diabetes and admission hyperglycemia predict COVID-19 severity by aggravating respiratory deterioration. Diabetes Res Clin Pract. 2020;168:108374.

8. Nyenwe EA, Kitabchi AE. The evolution of diabetic ketoacidosis: An update of its etiology, pathogenesis and management. Metabolism. 2016;65(4):507-21.

9. Kuchay MS, Reddy PK, Gagneja S, et al. Short term follow-up of patients presenting with acute onset diabetes and diabetic ketoacidosis during an episode of COVID-19. Diabetes Metab Syndr. 2020;14(6):2039041. 
10. Hollstein T, Schulte DM, Schulz J, et al. Autoantibody-negative insulin-dependent diabetes mellitus after SARS-CoV-2 infection: a case report. Nat Metab. 2020;2(10):1021-024.

11. Ghosh A, Anjana RM, Shanthi Rani CS, et al. Glycemic parameters in patients with new-onset diabetes during COVID-19 pandemic are more severe than in patients with new-onset diabetes before the pandemic: NOD COVID India Study. Diabetes Metab Syndr. 2020;15(1):215-20.

12. Siddiqui RS, Zirkiyeva M, Saliaj M. Onset of ketosis-prone diabetes in the setting of COVID-19 infection. Cureus. 2020;12(10):e10779.

13. Gustine JN, Jones D. Immunopathology of hyperinflammation in COVID-19. Am J Pathol. 2021;191(1):417.

14. Pradhan AD, Manson JE, Rifai N, et al. C-reactive protein, interleukin 6, and risk of developing type 2 diabetes mellitus. JAMA. 2001;286(3):327-34.

15. Martinez-Urbistondo M, Mora-Vargas A, Expósito-Palomo E, et al. Inflammatory-related clinical and metabolic outcomes in COVID-19 patients. Mediators Inflamm. 2020;25(2914275);1-7.

16. Zhu L, She Z-G, Cheng X, et al. Association of blood glucose control and outcomes in patients with COVID19 and pre-existing type 2 diabetes. Cell Metab. 2020;31(6):1068-077.

17. Hoffmann M, Kleine-Weber H, Schroeder S, et al. SARS-CoV-2 cell entry depends on ACE2 and TMPRSS2 and is blocked by a clinically proven protease inhibitor. Cell. 2020;181(2):271-80.

18. Fignani D, Licata G, Brusco N, et al. SARS-CoV-2 receptor Angiotensin I-Converting enzyme type 2 (ACE2) Is expressed in human pancreatic $\beta$-cells and in the human pancreas microvasculature. Front Endocrinol (Lausanne). 2020;11:596898.

19. Yang L, Han Y, Nilsson-Payant BE, et al. A human pluripotent stem cell-based platform to study SARSCoV-2 tropism and model virus infection in human cells and organoids. Cell Stem Cell. 2020;27(1):125-36.

20. Coate KC, Cha J, Shrestha S, et al. SARS-CoV-2 cell entry factors ACE2 and TMPRSS2 are expressed in the microvasculature and ducts of human pancreas but are not enriched in $\beta$ cells. Cell Metab. 2020;32(6):1028-040.

21. Wang F, Wang H, Fan J, et al. Pancreatic injury patterns in patients with coronavirus disease 19 pneumonia. Gastroenterology. 2020;159(1):369-70.

22. Tollard C, Champenois V, Delemer B, et al. An inaugural diabetic ketoacidosis with acute pancreatitis during COVID-19. Acta Diabetol. 2020:1-3.

23. Rubino F, Amiel SA, Zimmet P, et al. New-Onset Diabetes in Covid-19. N Engl J Med. 2020;383(8):78990. 